EPJ Web of Conferences 70, 00051 (2014)

DOI: 10.1051/epjconf/20147000051

(C) Owned by the authors, published by EDP Sciences, 2014

\title{
Unification with mirror fermions
}

\author{
George Triantaphyllou \\ ${ }^{1}$ National Technical University of Athens \\ 58 SINA Str., GR 10672 ATHENS, Greece, e-mail: gtriantaphyllou@aya.yale.edu
}

\begin{abstract}
We present a new framework unifying interactions in nature by introducing mirror fermions, explaining the hierarchy between the weak scale and the coupling unification scale, which is found to lie close to Planck energies. A novel process leading to the emergence of symmetry is proposed, which not only reduces the arbitrariness of the scenario proposed but is also followed by significant cosmological implications. Phenomenology includes the probability of detection of mirror fermions via the corresponding composite bosonic states and the relevant quantum corrections at the LHC.
\end{abstract}

\section{Introduction}

Attempts to unify fundamental interactions within a unique theory are not only based on aesthetic and philosophical grounds, but are also reinforced by experiments implying a convergence of the gaugecoupling strengths [1]. We report on previous work [2][3][4] explaining fermion family structure and the hierarchy between the electroweak (EW) symmetry scale and the coupling unification scale by introducing mirror fermions ("katoptrons"). Stabilization of the EW scale is achieved by using a new gauge interaction $S U(3)_{K}$ becoming strong around $1 \mathrm{TeV}$. The Higgs mechanism is based on mirror fermion condensates, similarly to, but differing significantly from, technicolor [5]. First, we inquire whether a particular breaking chain of an initial gauge symmetry $G=E_{8} \times E_{8}^{\prime}$ down to the Standard Model is compatible with the unification of the gauge couplings near the Planck scale and leads naturally to dynamical electroweak symmetry breaking at scales of around $1 \mathrm{TeV}$. Next, we incorporate the Lorentz group within $G$ in order to judge whether this approach is compatible with models of gravity based on spinors. Then, we justify the value of the gauge couplings at the unification scale in terms of a symmetry-group invariant. Last, a novel mechanism of symmetry emergence is proposed in order to reduce the arbitrariness of $G$ and discuss cosmological consequences.

\section{Coupling unification, symmetry breaking and spinor gravity}

One of the results of [2] is coupling unification, including the $S U(3)_{K}$ coupling. The starting point here is a different initial symmetry $G$ breaking at energies $\Lambda_{G U T}$ down to $S U(5) \times U(1)_{X} \times S U(5)^{\prime} \times$ $U(1)_{X}^{\prime} \times S U(3)_{K}$. We investigate the running of the gauge couplings in order to see if the energy scales involved have acceptable values. Under this symmetry, left-handed ordinary fermions $F$ and right-handed katoptrons $K$ transform as:

$$
\begin{aligned}
& F_{L}^{a}=(\overline{\mathbf{5}},-3, \mathbf{1}, 0, \mathbf{1})^{a} \oplus(\mathbf{1 0}, 1, \mathbf{1}, 0, \mathbf{1})^{a} \oplus(\mathbf{1}, 5, \mathbf{1}, 0, \mathbf{1})^{a} \\
& K_{R}=(\mathbf{1}, 0, \overline{\mathbf{5}},-3, \mathbf{3}) \oplus(\mathbf{1}, 0, \mathbf{1 0}, 1, \mathbf{3}) \oplus(\mathbf{1}, 0, \mathbf{1}, 5, \mathbf{3})
\end{aligned}
$$

This is an Open Access article distributed under the terms of the Creative Commons Attribution License 2.0, which permits unrestricted use, distribution, and reproduction in any medium, provided the original work is properly cited. 


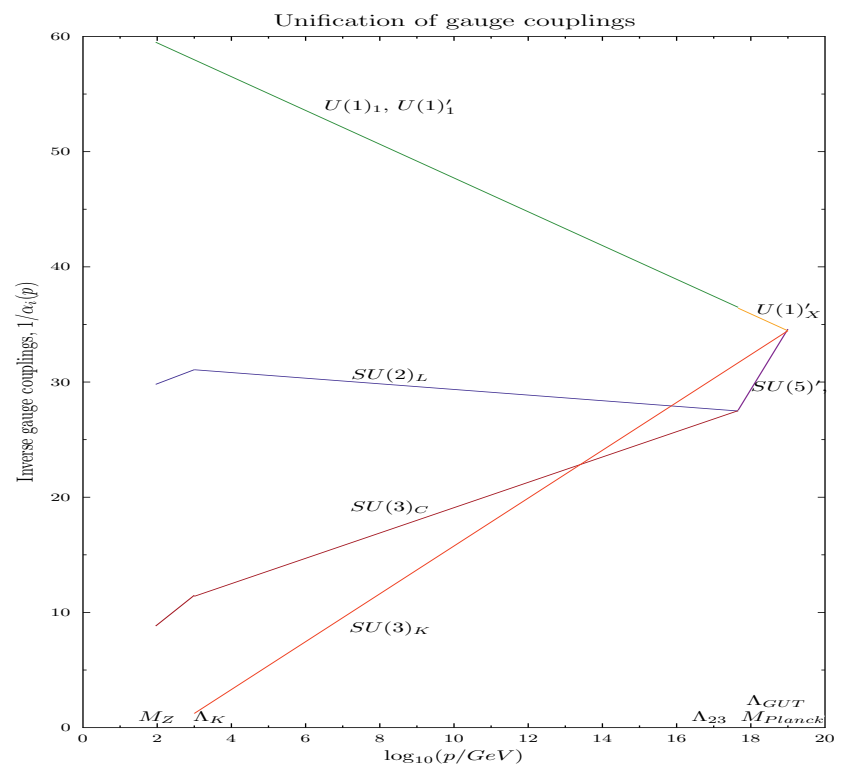

Figure 1. The renormalization of the gauge couplings at one loop [4]

where $a=1,2,3$ is a fermion generation superscript, 1 and 0 denote non-abelian and abelian group singlets respectively, ordinary fermions are singlets under $S U(5)^{\prime} \times U(1)_{X}^{\prime} \times S U(3)_{K}$ and appear in 3 generations, while katoptrons are singlets under $S U(5) \times U(1)_{X}$ and triplets under $S U(3)_{K}$, in an assignment inspired by "flipped $S U(5)$ " models [6].

The symmetry $S U(5) \times U(1)_{X} \times S U(5)^{\prime} \times U(1)_{X}^{\prime}$ is assumed to break at energies $\Lambda_{23}$ down to the Standard Model (SM) group times an abelian $U(1)_{1}^{\prime}$ felt only by katoptrons. One is then left with $S U(3)_{C} \times S U(2)_{L} \times U(1)_{1} \times U(1)_{1}^{\prime} \times S U(3)_{K}$, where $U(1)_{1}$ is the hypercharge group with a rescaled coupling. The fields leading to this breaking are neglected in the 1-loop calculation of coupling renormalization below. While katoptrons interact with the same $S U(3)_{C} \times S U(2)_{L}$ interaction as known fermions at energies below $\Lambda_{23}$, they carry their own $U(1)_{1}^{\prime}$ interaction down to the EW symmetry scale. Moreover, $S U(3)_{K}$ becomes strong near $\Lambda_{K}$, breaking itself and the EW symmetry [3]. The renormalization of the gauge couplings $g_{N}$ at energy scales $p$ for $N_{f}$ fermions in the fundamental representation of $S U(N)$ at 1-loop is given by $\alpha_{N}^{-1}(p)=\alpha_{N}^{-1}\left(p_{o}\right)+c\left(N, N_{f}\right) \ln \left(p / p_{o}\right)$, with $p_{o}$ some reference scale, $a_{N}=g_{N}^{2} / 4 \pi$ and $c\left(N, N_{f}\right)=\left(11 N-2 N_{f}\right) / 6 \pi$. The katoptron coupling $\alpha_{K}$ evolves at scales ranging from $\Lambda_{K}$ to the unification scale $\Lambda_{G U T}$ according to $c(3,8)=17 / 6 \pi$. The $S U(2)_{L}$ coupling $\alpha_{2}$ and the $S U(3)_{C}$ coupling $\alpha_{3}$ evolve according to $c(N, 12)=(11 N-24) / 6 \pi$ at energies where both ordinary and katoptron fermions contribute to the beta functions, i.e. between $\Lambda_{K}$ and $\Lambda_{23}$. Either below $\Lambda_{K}$, or when fermions and katoptrons interact with distinct groups, as is the case for all the $U(1)$ and the $S U(5), S U(5)^{\prime}$ couplings, couplings evolve according to $c(N, 6)=(11 N-12) / 6 \pi$, with 
$N=0$ for the $\alpha_{X}, \alpha_{X}^{\prime}, \alpha_{1}, \alpha_{1}^{\prime}$ couplings of $U(1)_{X}, U(1)_{X}^{\prime}, U(1)_{1}, U(1)_{1}^{\prime}$ respectively, and $N=2,3,5$ for the $S U(2)_{L}, S U(3)_{C}$ couplings and the $\alpha_{5}, \alpha_{5}^{\prime}$ couplings of $S U(5), S U(5)^{\prime}$ respectively. Taking into account the relevant boundary conditions and noting that $\alpha_{K}\left(\Lambda_{K}\right) \sim 1$ and $M_{Z} \sim 91.2 \mathrm{GeV}$, we estimate $\Lambda_{G U T}, \Lambda_{23}$, and $\Lambda_{K}$, assuming a "big desert" between these scales. In particular, we find (see Figure 1) [4]: $\Lambda_{G U T}=M_{Z} \times 10^{17} \sim 10^{19} \mathrm{GeV} \sim$ M $_{\text {Planck }}, \Lambda_{23}=M_{Z} \times 6 \times 10^{15} \sim 5 \times 10^{17} \mathrm{GeV}$ and $\Lambda_{K}=M_{Z} \times 11 \sim 1 \mathrm{TeV}, \alpha\left(\Lambda_{G U T}\right) \sim 0.029$ and $\alpha_{3}\left(\Lambda_{23}\right) \sim 0.036$. Moreover, we see that $\Lambda_{K} \sim M_{\text {Planck }} \exp \left(-\frac{6 \pi}{17 \alpha\left(\Lambda_{G U T}\right)}\right)$, a relation rendering transparent the solution of the hierarchy problem. Furthermore, the value of $\Lambda_{23}$ renders this scenario most probably safe with regards to proton decay.

Next, the symmetry breaking chain from a group $G$ down to $S U(5) \times U(1)_{X} \times S U(5)^{\prime} \times U(1)_{X}^{\prime} \times$ $S U(3)_{K}$ and then down to the SM is explored using [7]. Non-zero vacuum expectation values (vevs) of effective composite fields are taken to lead to the breaking channels needed. These are fermion condensates arising non-perturbatively to safeguard gauge invariance at tree level. Denoting as $L G$ the symmetry $S U(2) \times S U(2)^{\prime}$, we assume that the following symmetry breaking chain is obtained, triggered by the antisymmetric $\mathbf{2 4 8}_{a}$ condensate and starting from $M_{\text {Planck }} \sim \Lambda_{G U T}$ [4]:

$$
\begin{array}{r}
E_{8} \times E_{8}^{\prime}\left(\text { at } \Lambda_{\mathrm{GUT}}\right) \rightarrow \\
S U(5) \times U(1)_{X} \times S U(5)^{\prime} \times U(1)_{X}^{\prime} \times S U(3)_{K} \times L G\left(\text { at } \Lambda_{23}\right) \rightarrow \\
S U(3)_{C} \times S U(2)_{L} \times U(1)_{1} \times U(1)_{1}^{\prime} \times S U(3)_{K} \times L G\left(\text { at } \Lambda_{\mathrm{K}}\right) \rightarrow \\
S U(3)_{C} \times U(1)_{e m} \times L G
\end{array}
$$

To proceed, we argue that $S U(2) \times S U(2)^{\prime} \approx L G$ contains the Lorentz symmetry $S O(3,1)$ (up to discrete subgroups), an assumption motivated by the proximity of $\Lambda_{G U T}$ to $M_{\text {Planck }}$. Formally, one should decompose the $E_{8}$ above as $E_{7}(C) \times S L(2, C) \subset E_{8}(C)$ [8]. One then gets from the two $E_{8} \mathrm{~s}$ the group $S O(4, C) \approx S U(2, C) \times S U(2, C)^{\prime}$ ( since $S U(2, C) \approx S L(2, C)$ ) which has both $S O(4)$ and $S O(3,1)$ as subgroups. The $L G$ group is therefore assumed to contain the Lorentz group and it is taken to be a global, not a local symmetry. In order to render the relation between $L G$ and the Lorentz group consistent, one needs to equate the $S O(3,1)$ coupling with the gravitational coupling. Problems related to the Coleman-Mandula theorem [9] are discussed in [4]. Within a framework similar to [10], we consider a metric of the form $g_{\mu \nu}=E_{\mu}^{m}(x) E_{v}^{n}(x) \eta_{m n}=E_{\mu}^{m}(x) E_{v m}(x)$, where $\mu, v=0, \ldots, d$ are spacetime indices, $m, n=0, \ldots, d$ are indices corresponding to the "internal" Lorentz symmetry with $\eta_{m n}=\operatorname{diag}(-1,1, \ldots, 1)$, while $E_{\mu}^{m}(x)=<\tilde{E}_{\mu}^{m}(x)>\sim \delta_{\mu}^{m} M_{\text {Planck }}$ for $\mu, \mathrm{m}=0, \ldots, 3$ and $E_{\mu}^{m}(x)=<$ $\tilde{E}_{\mu}^{m}(x)>\sim 0$ for $\mu, \mathrm{m}=4, \ldots, \mathrm{d}$ are soldering forms (vielbeins), i.e. vevs of operators $\tilde{E}_{\mu}^{m}(x)$ breaking the Lorentz symmetry spontaneously. Symmetric fluctuations of such a metric around the Minkowski spacetime are expected to produce Goldstone bosons identified with gravitons [10] [11]. In the spinor gravity approach [11], these vevs have a dynamical origin since they are expressed as vevs of fermion bilinear operators $\tilde{E}_{\mu}^{m}(x)=\frac{i}{2}\left\{\bar{\Psi}(x) \gamma^{m} \partial_{\mu} \Psi(x)-\partial_{\mu} \bar{\Psi}(x) \gamma^{m} \Psi(x)\right\}$, where $\gamma^{m}$ are Dirac matrices in d dimensions and $\Psi, \bar{\Psi}$ are Grassmann variables. A relevant partition function, effective action and effective potential can then be formally defined, and these are expected to lead, in lowest order in the effective potential expansion, to equations similar to the ones of General Relativity [11], in a way that spacetime is not treated as background but is incorporated in the equations non-perturbatively. In such a picture, physical distances are induced by fermion correlation functions and the appearance of a metric is inherently quantum-mechanical. To make connection with our model, we use the unification symmetry $E_{8} \times E_{8}^{\prime}$, adopting the fermion-bilinear approach for the soldering forms [11] in order to maintain the dynamical interpretation of the breaking of $G=E_{8} \times E_{8}^{\prime}$, where internal dimensions are connected with the appearance of gauge symmetries in $4 \mathrm{~d}$. Non-zero vevs of antisymmetric fermion bilinears sitting in the $\mathbf{2 4 8}_{a}$ of $E_{8}$ and $E_{8}^{\prime}$ are expected to lead to the breaking sequence needed. The $S U(2)$ triplets in $(\mathbf{1 , 3})$ and doublets in $(\mathbf{5 6 , 2})$ contained in the decompositions of the $\mathbf{2 4 8}_{a}$ of each of 
the $E_{8}, E_{8}^{\prime}$ under $E_{7} \times S U(2)$ can break spontaneously $L G$ after acquiring non-zero vevs, leading thus to a dynamically generated metric, assuming that $\left\langle\bar{\Psi} \gamma^{m} \partial_{\mu} \Psi>=0\right.$ for $m>3$. The vevs of fields in the $(1,3)$ of $E_{7} \times S U(2)$ and $E_{7}^{\prime} \times S U(2)^{\prime}$ constitute the most attractive antisymmetric channel of $E_{8} \times E_{8}^{\prime}$ breaking down to $E_{7} \times E_{7}^{\prime}$, possibly justifying their dominance in determining spacetime dimensionality over the $(\mathbf{5 6 , 2})$ and $\left(\mathbf{1 3 3 , 1 )}\right.$ vevs breaking subsequently $E_{7} \times E_{7}^{\prime}$ and the remnant of $L G$. In any case, note that the same type of fermion condensate is responsible not only for metric generation but for gauge symmetry breaking as well.

In order for $L G$ to break down to its diagonal subgroup $S O(3,1)$, one could consider vevs of 4-fermion composite operators, corresponding to gauge-invariant terms in the initial action and transforming as $\left(\mathbf{1 , 3 , 1 , 3 )}\right.$ under $E_{7} \times S U(2) \times E_{7}^{\prime} \times S U(2)^{\prime}$. One way to couple the two initial -in principle decoupled- $E_{8}$ sectors in a way having the same end result as such operators is to consider an initial symmetry of the form $E_{8} \times E_{8}^{\prime} \times\left(Z_{2}^{E_{8}} / Z_{2}^{S U(2)}\right)$, where the discrete $Z_{2}$ symmetries interchange the two $E_{8}$ groups and their $S U(2)$ subgroups respectively, in a construction similar to [12][13]. A deeper analysis of this scenario might have the potential of not only explaining the dimensionality of our space-time, but its signature as well, i.e. why the group $S O(3,1)$ emerges instead of $S O(4)$ or $S O(2,2)$ for instance. We then consider the effective fermionic action in $4 \mathrm{~d}$ in a derivative and fermion-field expansion to 1-loop: $S_{\text {eff }} \sim \int d^{4} x \operatorname{det}\left(E_{\mu}^{m}(x)\right)\left(c_{1}+c_{2} R+c_{3} \bar{\Psi}(x) \gamma^{m} E_{m}^{\mu}(x) D_{\mu} \Psi(x)+\ldots\right)$ plus gauge kinetic terms, where $\operatorname{det}\left(E_{\mu}^{m}(x)\right)=\sqrt{\operatorname{det}\left(-g_{\mu \nu}\right)} \neq 0$ while $\mu, v, m=0, \ldots, 3, c_{1,2,3}$ are constants and $D_{\mu}$ is the gauge-covariant derivative corresponding to the $E_{7} \times E_{7}^{\prime}$ gauge symmetry after compactification. This symmetry should probably be corrected by a multiplicative factor of the form $\left(Z_{2}^{E_{7}} / Z_{2}^{S U(5)}\right)$ interchanging the two $E_{7}$ groups and their respective $S U(5)$ subgroups in a way that couples the right- and left-handed sectors of the theory during compactification causing the breaking of $S U(5) \times S U(5)^{\prime}$ to their diagonal subgroup at $\Lambda_{23}$ [4]. The first term of the action above gives a cosmological constant $\Lambda$, the second the Ricci curvature, and the third the action in [10]. The result above is expected to stem from an action expressed as $S_{f} \sim \int d^{d} x \operatorname{det}\left(\tilde{E}_{\mu}^{m}\right)$ before compactification.

We then investigate the dimensionality $d$ of space to integrate our Lagrangian over. Naturalness reasons lead us to consider the action as an integral over a manifold having the isometry $E_{8} \times E_{8}^{\prime}$ up to discrete factors. The proper number of (complex) dimensions is then $d=16$, equal to the number of roots of the groups involved. Integrating over the 14 extra internal dimensions gives us $E_{7} \times E_{7}^{\prime}$, which by the way correspond to 2 lattices formed by the unit-norm imaginary Caley octonions, and should leave us with 2 complex, i.e. 4 real, ordinary spacetime dimensions. The internal dimensions are then assumed to be compactified at a size of around $1 / M_{\text {Planck }}$ to avoid the appearance of Kaluza-Klein excited states at energies lower than $M_{\text {Planck. }}$. It is conjectured below that the space with the isometries needed is the quotient space of the $16 \mathrm{~d}$ maximal torus $T^{16}$ by the lattice $\pi \Gamma_{8} \times \pi \Gamma_{8}$ generated by the roots of $E_{8} \times E_{8}^{\prime}$ multiplied by integer multiples of $\pi$. In the following, an effort is made to motivate further our choice of $E_{8} \times E_{8}^{\prime}$ as a unification symmetry group based on the special packing-density and kissing-number properties of the $E_{8}$ lattice, in addition to its self-duality feature which might prove to be unique and crucial for unifying spacetime with gauge symmetries. The $8 \mathrm{~d}$ spaces of the two $E_{8}$ groups have to be treated distinctly, since, while each of the two $E_{8}$ lattices provide the highest kissing (coordination) number and densest sphere packing in $8 \mathrm{~d}$, in $16 \mathrm{~d}$ other lattices like the Barnes-Wall (BW) lattice provide a higher kissing number and denser sphere packing than $\Gamma_{8} \times \Gamma_{8}$, even though they are not self-dual. In particular, the $\Gamma_{8} \times \Gamma_{8}$ kissing number equals 480 , while the BW lattice has a kissing number equal to 4,320. However, since lattices like BW do not correspond to any root system, they cannot generate the symmetries needed. In general, densest sphere packings in dimensions $d>8$ are most likely either disordered, not corresponding to lattices, or the lattices they correspond to are not associated with root systems and thus known gauge symmetries. Therefore, all other cases for $d>8$ cannot lead, on the basis of such arguments, to the symmetries needed. A related 
argument supporting this scenario is based on the concept of optimal lattices [4] [14]. In any case, the above might be an indication that we are approaching a theory starting from an action as simple as the one above and yielding the dimension not only of spacetime but also of the internal space and its isometries by the dynamics of the action itself, without having to postulate them a priori.

\section{Critical behaviour and emergence of symmetry}

In order to connect our action to the results above, one needs first to justify the value of $\alpha\left(\Lambda_{G U T}\right)$ calculated in section 2. We are dealing with a critical phenomenon breaking $E_{8} \times E_{8}^{\prime}$ and having as order parameters the non-zero vevs related to the metric. The relevant critical parameters are the couplings of the two $E_{8} \mathrm{~s}$. The order parameters are assumed here to scale as $E_{\mu}^{m} \sim \frac{p^{m} p_{\mu}}{M_{\text {Planck }}}<\bar{\Psi} \Psi>$ with fermions $\Psi$ in the fundamental representations of $E_{8}$ and $E_{8}^{\prime}$ and $\left|p^{m}\right|,\left|p_{\mu}\right| \sim M_{\text {Planck }}$. The $<$ $\bar{\Psi} \Psi>$ condensate, corresponding to the most attractive channel, is assumed to be the catalyst for the formation of the antisymmetric condensates in the $\mathbf{2 4 8}_{a}$ of the two $E_{8} \mathrm{~s}$. Since $E_{8} \times E_{8}^{\prime}$ breaks at around $M_{\text {Planck }}$, the critical coupling $\alpha_{S B}$ is estimated by a modified Nambu-Jona-Lasinio formalism yielding $\alpha_{S B} \sim C_{2}^{-1} \sim 0.03 \sim \alpha\left(\Lambda_{G U T}\right)$, with $C_{2}=30$ the quadratic Casimir invariant of $E_{8}$ [4]. The wide hierarchy between $M_{\text {Planck }}$ and the weak scale can then be traced back to the magnitude of $C_{2}$, since $\Lambda_{K} \sim M_{\text {Planck }} \exp \left(-1.23 C_{2}\right)$.

We now examine the dynamics leading to the emergence of symmetry by applying techniques borrowed from solid-state physics, chemistry, biology and even sociology, the common starting ground being the emergence of configurations exhibiting spontaneous self-organization transitions in ordered structures and nucleation, like DNA, neural networks or crystals, i.e. processes characterized by "selforganised criticality" [15]. We place our action on a lattice to see if its qualitative behaviour can be inferred by simpler or similar systems in lattice gauge theories or solid-state systems. The effective action $S_{l a t}$ to lowest order, apart from the Einstein-Hilbert terms, is written as $S_{l a t}=\sum_{<i, j>} \mathcal{E}_{i j} \bar{\Psi}_{i} \Psi_{j}$, where $\mathcal{E}_{i j}$ is an antisymmetric matrix proportional to the system's volume and encoding information on $E_{\mu}^{m}$, and the sum over the lattice sites $i, j$ is restricted over nearest neighbours.

In order to study the emergence of the $E_{8}$ lattices from first principles, we use a model suitable for percolation phenomena, a "toy" model in our case, assumed here to belong to the same universality class, i.e. the single-state $(q=1)$ Potts model, with Hamiltonian given by $H_{P}=-J \sum_{<i, j>} \delta\left(S_{i}, S_{j}\right)$, where $J>0$ is the coupling strength and $\delta\left(S_{i}, S_{j}\right)=1$ when $S_{i}=S_{j}=1$ and zero otherwise. The partition function $Z=\sum_{C_{i}} e^{-\beta H_{P}}$ is given by

$$
Z=\sum_{C_{i}}\left(e^{\beta J}-1\right)^{E_{i}}
$$

where the sum is over clusters $C_{i}$ consisting of $E_{i}$ edges and $1 / \beta=k_{B} T$. Here, the occupation probability $p$ is given by $p=1-e^{-\beta J}$ and increases with decreasing $T$. Such discrete models are usually studied on lattices with given dimensionality $d$ and coordination (kissing) number (number of nearest neighbours) $c$. In the following, we explore the behaviour of a system of nodes minimizing its free energy by adjusting its $d$ and $c$ in order to form an optimal lattice. For high $T, w \equiv e^{\beta J}-1 \sim$ $p \sim \beta J \ll 1$ in equation 3 implies that only clusters with few edges contribute significantly to the partition function. In a competition between annihilation and aggregation of large clusters, those of low $d$ and $c$ dominate. This system of nodes, a rough model of the "pre bib-bang" world, lies initially in a highly-probable state, 1.e. having large $T$ and entropy $S$, obviating the need for contrived cosmological boundary conditions. Evolution is dictated by the system's need to reduce its energy, which is achieved by lowering $T$ and expanding. This irreversible gradual process might define an 
"arrow of time", the increase in $S$ compensating the entropy loss due to the formation of the spacetime "crystal", providing an explanation of the 2 nd law of thermodynamics.

There is a certain $T$ however for which the behaviour of the partition function $Z$ in equation 3 changes dramatically. This change proves to be crucial for our argument towards symmetry emergence. For low $T$ such that $k_{B} T \leq k_{B} T_{c}=J / \ln 2 \sim 1.4 J$, we find $p \geq 1 / 2$ and $w \geq 1$, implying that large clusters consisting of many edges dominate over the smaller ones! Those familiar with the q-state Potts model recognize in this expression $T_{c}$ given by $e^{\beta_{c} J}=(1+\sqrt{q}) / \sqrt{q}$ for $q=1$. This has a highly non-trivial and far-reaching impact on the topology of the network of nodes. Since for a network to have any sense we assume that $\min \left(E_{i}\right)=c$, a lattice like the one of $E_{8}$ with $c=240$ contributes much more to the partition function than the conventional $\mathcal{Z}^{8}$ lattice having $c=16$. Approaching $T_{c}$ amounts therefore to having a $E_{8}$ lattice emerge spontaneously from the dynamics. We leave for future investigations the study of how a more complicated variant of this lattice, like the $E_{8} \times E_{8}^{\prime} \times\left(Z_{2}^{E_{8}} / Z_{2}^{S U(2)}\right)$ appearing here, possibly with the extra factor $\left(Z_{2}^{E_{7}} / Z_{2}^{S U(5)}\right)$ arising during compactification, finally emerges. The relevance of densest-sphere packing and "highest kissing number" arguments presented in the previous section is now apparent, since this particular lattice offers an optimal configuration with regards to $c$ and might be preferred over alternative arrangements not offering so many edges per node. Note moreover that "crystal" clusters with $E_{i}=240$ evolve even when $T>T_{c}$. However, such clusters should lie within limited regions. Moreover, at $T=T_{c}$ we expect a latent heat, or enthalpy, given by $H=478 \mathrm{~J}$ [4]. A more careful calculation of enthalpy might produce a smaller value for $H$ due to the action of the $Z_{2}$ discrete symmetries.

At this critical point other lattices with even higher $d$ and $c$ could also form. These however do not lead to the symmetries observed in our world, as explained in the last section. This implies that we might be living within a metastable region, with other Universe domains corresponding to different configurations of lattice points, devoid of the known interactions. This is consistent with Ostwald's rule in polymorphic and allotropic crystallography, according to which the least stable polymorphs crystallize first, leading to transformations between closest phases with regards to free energy. Metastability of the two $E_{8}$ lattices is central to the argument presented, should be studied more thoroughly, and is at least qualitatively supported from the fact that these lattices offer a local maximum for a properly-normalized kissing number $(\mathrm{nKN})$ and for the centre density, defined as the ratio of sphere-packing density over the unit-sphere volume for certain dimensions $d$. Indeed, the $E_{8}$ lattice offers a centre density higher than the maximum achievable for $8<d<12$ [16]. In parallel, regarding known sphere packings, it offers maximal centre density for $6<d<18$ and a local maximum for $\mathrm{nKN}$ (see Figure 2) [17]. The next dimension probably providing a local maximum for the centre density and for nKN hosts the even unimodular (i.e. self-dual) Leech lattice in $d=24$ offering maximal centre density for $0<d<28$ and a kissing number equal to 196,560, exhibiting symmetries not associated with the known Lie groups. (Instead, it is closely connected to the largest sporadic finite simple group.) Such lattices $\Gamma$ are usually studied via theta functions defined as $\Theta_{\Gamma}(z)=$ $\sum_{\lambda \in \Gamma} e^{i \pi z|\lambda|^{2}}(\operatorname{Im}(\mathrm{z})>0)$, which are modular forms of weight $d / 2$ if $\Gamma$ is even and self-dual, which implies that $d=0 \bmod (8)$.

Next, we describe some potentially interesting cosmological implications of this critical behaviour. We take the measured $\Lambda \sim 2 \times 10^{-3} \mathrm{eV}$ to correspond to the free energy of a minimal cluster of the $E_{8} \times E_{8}^{\prime}$ lattice having 240 edges from each of the two $E_{8}$ 's, i.e. $E_{i}=480$, and $T_{C B R} \sim 2.7 \times$ $10^{-4} \mathrm{eV}$ of the cosmic background radiation to be equal to the system's temperature. Then, the critical free-energy expression yields $\Lambda \sim 7 k_{B} T_{C B R} \sim 10 J$ and $\epsilon \equiv\left(T_{C B R}-T_{c}\right) / T_{c} \sim 1 \%$ [4]. This implies a certain fine-tuning close to criticality for $T$. Taking the Universe to be in a "glass-tocrystal" transition, typical relaxation and equilibration times $\tau$ for glassy dynamics are huge compared to the microscopic ones of ferromagnetic-type systems. This leads us to considering non-adiabatic 
ICFP 2012

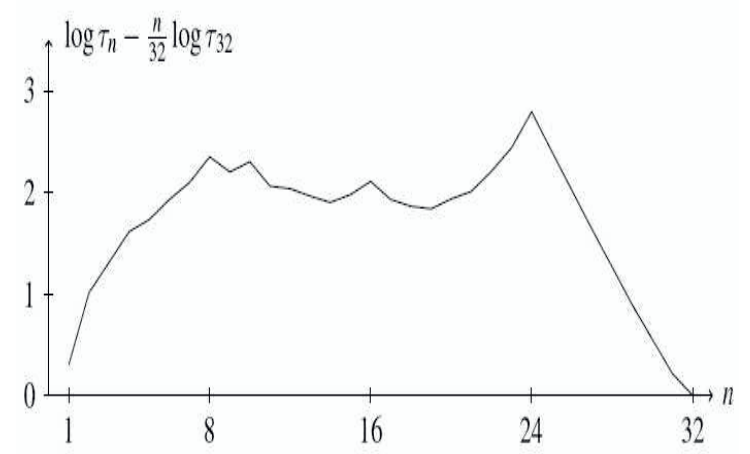

Figure 2. A function (nKN) of the maximum lattice kissing number $\tau_{n}$ in $R^{n}$ for $0<n<33$ [17]

phenomena where relaxation times are of cosmological scale, and we might be living within such a critical period. Another issue to address is the smallness of $\Lambda$ in comparison to $M_{\text {Planck }}$. The solution might be coming from the Hamiltonian we started with, which includes only local, nearestneighbour, interactions, effectively introducing a very large infrared cut-off. One may introduce a phenomenological potential $V(r)$ between lattice sites being separated from each other by a distance $r$ given by $V(r)=-2 E_{i} J\left(\frac{L_{\text {Planck }}}{r}\right)^{d-3}\left(1-\frac{\sqrt{J a}}{2}\left(\frac{a}{r}\right)^{d}\right)$, with $L_{\text {Planck }}=1 / M_{\text {Planck }}, d=16$ and $a$ having dimensions of length corresponding to the distance where "repulsive" effects become important. For large $r, V(r)$ vanishes like $1 / r^{d-3}$, since the 2-point correlation function in position space falls as $1 / r^{d-2}$. Using the relation $J L_{\text {Planck }}=\left(\frac{L_{\text {Planck }}}{a}\right)^{2 d+1}$ and taking $a \sim 9.3 L_{\text {Planck }}$ gives the required hierarchy $\Lambda \sim 10^{-31} M_{\text {Planck. }}$.

Consequently, a higher-dimensional analogue of spin-glass phase transitions might provide a picture for the emergence of $E_{8} \times E_{8}^{\prime}$ at the beginning of our Universe, as a kind of "liquid-to-solid", freezing phase transition, or a kind of disorder-order, "glass-to-crystal" transition. Regarding entropy $S$, the only way for the system to compensate for the loss of $S$ within a spacetime volume Vol during a time $d t$ is to expand, changing its volume by $d(V o l)$. We note that large values for $E_{i}$ imply a period resembling inflation. Other cosmological implications include the existence of macroscopic domains in the Universe not having the symmetries observed in our neighbourhood. Particles within such regions would not interact in familiar ways, for instance not feeling electromagnetic interactions and supplying an explanation for Dark Matter (DM). The luminous parts of galaxies would occupy regions corresponding to the "jammed", ordered phase of spacetime, domain states of ferromagnetic type, like "crystal bubbles" within a glass-type, amorphous spacetime structure. For a solid-state Physics analogy in the interdisciplinary spirit of this Conference, compare the galaxy distribution within DM regions (for instance, see relevant pictures from NASA, ESA, C. Heymans, M. Gray, STAGES Coll.(2008) ) with quartz (silicon crystal) distribution within silicon glass regions (see for instance M. Chen, W. Xiao, X. Xie, E. Plan. Sc. Lett. 297 (2010) 306). An order-of-magnitude estimate of the ratio $R$ of crystal-to-glass-type volumes is given by $R=1-\exp \left(-\Delta F / k_{B} T\right)$, where $\Delta F$ is the free energy gained by the system by being in the "crystal" state. It is possible that "spacetime" nucleation continues today, implying a growth of the luminous-to-DM ratio on cosmological timescales. A detailed relevant analysis would allow the prediction of galactic DM concentrations and of 
the average structure of the underlying spacetime lattice. It would allow answering questions like "is the structure of spacetime within the intergalactic voids of glass- or crystal-type?". Assuming that all "crystal" domains are occupied by visible galaxies implies that $R \sim 5 \%$, while taking intergalactic voids to be also "crystal"-like raises $R$ to around 77\%. Other possibilities include DM regions corresponding either to alternative $E_{7}$ symmetry breakings, or to denser "sphere packings" not linked to a group's root system. The latter would imply that our vacuum has already started decaying towards a more stable configuration like the Leech lattice, leading to growing DM and shrinking luminous domains.

Alternatively, in off-equilibrium phenomena of crystal and glass formation, the fluctuationdissipation theorem in fast transitions is violated, since the system does not have enough time to relax to its new equilibrium, forcing us to consider "effective temperatures" $T_{\text {eff }}$ larger than the heatbath ones [18]. Here, $R \sim 50 \%$ implies $T_{\text {eff }} \sim 10 T_{C B R}$, while $R \sim 80 \%$ implies $T_{\text {eff }} \sim 5 T_{C B R}$. If the universe expanded and cooled too fast to have relaxed to equilibrium, $T_{\text {eff }}$ for "crystal" formation is larger than $T_{C B R}$. Such values of $T_{\text {eff }}$ are consistent with treating particles as topological or vacancy defects on the lattice background described above, analogous to positively-charged holes in an electron sea or lattice, their number density $d_{p}$ in the Universe being a function of enthalpy cost and approximately equal to the ratio of their entropy $S_{p} \sim k_{B} 10^{89}$ to the Universe entropy $S_{B} \sim k_{B} 10^{122}$ assuming a Bekenstein-bound saturation [19], 1.e. $d_{p} \sim S_{p} / S_{B} \sim 10^{-33} \sim \exp \left(-H / k_{B} T_{\text {eff }}\right)$ implying that $T_{e f f} \sim 4.4 T_{C B R}$. This result favours the characterization of intergalactic voids as "crystal-like". Note that the discrepancy between $T_{C B R}$ and $T_{\text {eff }}$ might be smaller if a more careful calculation of $H$ is performed. This approach has far-reaching implications on structure formation, consistent with the view that stars are born within DM halos. It might lead to an understanding of the shape of spiral galaxies on the basis of "helicoidal dislocations" in crystals. It might explain the large voids between galactic clusters, which are usually found to be larger than what simulations suggest, since crystals usually displace vacancies and other impurities towards boundaries of different phases and form vacancy clusters to minimize their energy. Here, the role of impurities is played by DM "amorphous" regions containing small crystal "islands", i.e. galaxies, full of vacancies corresponding to elementary particles. For a solid-state Physics analogy in the interdisciplinary spirit of this Conference, compare the galaxy number density field (see for instance W. Shaap, PhD Thesis, U. Groningen 2007) with vacancy clusters in copper (see for instance M. Kiritani, Jour. Nucl. Mat. 276 (2000) 41). Future experiments could probe the spacetime structure within DM domains, or measure the energy release, perhaps in the form of, hitherto unexplained, ultra-high energy cosmic rays, when the "crystal" forms.

Next, we discuss briefly quantization of our action. By having $\Gamma_{8} \times \Gamma_{8}^{\prime}$ emerge with lattice spacing equal to $L_{\text {Planck }}$, one achieves a cellular decomposition of spacetime with a UV cut-off equal to $M_{\text {Planck }}$ avoiding singularities plaguing quantum gravity. This might have a dramatic impact not only on the general renormalization programme but also on black holes, the initial singularity of spacetime and gravitational collapse, analogous to the false prediction of atom collapse before the advent of quantum mechanics. In an approach close to spin networks and lattice Yang-Mills, we have lattice nodes corresponding to $4 \mathrm{~d}$ spacetime points, a "world crystal". On each node there is a fiber corresponding to $E_{7} \times E_{7}^{\prime}$ stemming from 14 compactified dimensions. This provides an understanding of Heisenberg's uncertainty principle, since a particle, seen as a vacancy defect, extends at least between 2 adjacent nodes, reminding us of Wheeler considering particles as "quantum geometrodynamic excitons" [20]. Experiments around $M_{\text {Planck }}$ should distinguish such a spacetime fabric from models treating particles as extended objects on a continuous background. Ideas along these lines might include far in the future a gravitational analogue of Bragg spectroscopy probing the microstructure of spacetime. 


\section{Discussion}

A phenomenological issue related to the fermion content considered here is that it is usually associated with a large $S$ parameter. However, it is possible that vertex corrections can drive the $S$ parameter close to zero [21]. On the other hand, LEP and Fermilab results at the $2-3 \sigma$ level suggest massdependent anomalous quark couplings [22] and an excess of dijet plus W events [23], signals compatible with quark-katoptron mixing and katoptron bound states. Moreover, assuming that this theory is correct, recent LHC results regarding a new boson having a mass of around $125-6 \mathrm{GeV}$ correspond to the lightest member of a series of katoptronic mesons analogous to QCD or technicolor mesons. This lightest meson, a "katoptronic pion" corresponding to the lightest katoptrons, is expected to have comparatively very small couplings to third-generation ordinary fermions, like the bottom and top quark, due to the comparatively small mixing of the lightest katoptrons to third-generation ordinary fermions [24]. To conclude, while waiting for new relevant results from the LHC, we exposed an attempt to unify gauge with gravitational interactions using $E_{8} \times E_{8}^{\prime}$ emerging naturally from first principles. It leads to coupling unification and to an understanding of the unification coupling strength from an invariant of the emergent symmetry group; it reproduces the symmetries, the family structure of matter and the dimensionality of spacetime, not treating it as background; it solves the hierarchy problem between the Planck, the weak and the cosmological constant scale. Moreover, it exhibits a unique vacua sequence with cosmological implications like the interpretation of DM as having a topological origin. Securing the above on a firm basis needs a new physical principle which is more fundamental than a given spacetime or gauge symmetry and lies presumably at the heart of several other scientific areas as well (like crystallography, cognitive science etc.); according to it, spacetime, matter, and their symmetries, emerge naturally from a set of identical, distinct elementary fields connected to each other optimally.

\section{References}

[1] J.Pati, A.Salam, Phys. Rev. D10 (1974) 275; H.Georgi, S.Glashow, Phys. Rev. Lett. 32 (1974) 438.

[2] G. Triantaphyllou, Eur. Phys. J. C10 (1999) 703.

[3] G. Triantaphyllou, Mod. Phys. Lett. A16 (2001) 53.

[4] G. Triantaphyllou, Electron. J. Theor. Phys. 10 (2013) 28, 135.

[5] S. Weinberg, Phys. Rev. D13 (1976) 974; L. Susskind, Phys. Rev. D20 (1979) 2619.

[6] S.M. Barr, Phys. Lett. B112 (1982) 219.

[7] R. Slansky, Phys. Rept. 79 (1981) 1.

[8] See for instance J. Distler, S. Garibaldi, Commun. Math. Phys. 298 (2010) 419.

[9] S.R. Coleman, J. Mandula, Phys. Rev. 159 (1967) 1251.

[10] R. Percacci, Phys. Lett. B144 (1984) 37.

[11] A. Hebecker, C. Wetterich, Phys. Lett. B574 (2003) 269;

[12] G. Triantaphyllou, G. Zoupanos, Phys. Lett. B489 (2000) 420.

[13] P. Forgacs, Nucl. Phys. 308 (1988) 477.

[14] H. Cohn, A. Kumar, Jour. Am. Math. Soc. 20 (2007) 99.

[15] S. Torquato, F.H. Stillinger, Rev. Mod. Phys. 82 (2010) 2633.

[16] H. Cohn, N. Elkies, Ann. Math. 157 (2003) 689.

[17] H. Cohn, Y. Jiao, A. Kumar, S. Torquato, Geom. Topol. 15 (2011) 2235.

[18] D. Herisson, M. Ocio, Eur. Phys. J. B40 (2004) 283.

[19] C.A. Egan, C.H. Lineweaver, Astroph. Jour. 710 (2010) 1825. 
[20] G. Preparata, S. Rovelli, S.S. Xue, Gen. Rel. Grav. 32 (2000) 1859.

[21] G. Triantaphyllou, Int. J. Mod. Phys. A15 (2000) 265.

[22] P.B. Renton, Eur. Phys. J. C8 (1999) 585; CDF Collaboration, Phys. Rev. D83 (2011) 112003.

[23] CDF Collaboration, Phys. Rev. Lett. 106 (2011) 171801.

[24] G. Triantaphyllou, J. Phys. G26 (2000) 99. 